\title{
Implementing Office-Based Opioid Treatment Models in Primary Care
}

\author{
E. Marshall Brooks, PhD and Sebastian Tong, MD, MPH
}

Background: Primary care practices are ideal sites for integrating medication-assisted treatment (MAT) for opioid use disorder, but little is known about how practices have achieved this. Our study aimed to describe the implementation experiences and treatment models of practices implementing MAT.

Methods: We conducted a qualitative analysis of MAT integration at 26 practices across Virginia after the state implemented the Addiction and Recovery Treatment Services (ARTS) benefit in 2017. Data collection activities included interviews with clinic team members, including buprenorphine-waivered prescribers, behavioral health clinicians, care coordinators, and peer counselors. We used a template analysis approach to thematically analyze data.

Results: Our study identified various ways in which MAT can be implemented in primary care clinics and other ambulatory settings. Although state regulations and treatment guidelines suggest colocating behavioral health counseling and medication management, providing care coordination, and conducting regular urine drug screens, we found a wide spectrum of ways in which practices can adapt and innovate treatment models to fit local needs.

Discussion: As the fight against the opioid epidemic continues, we need to identify feasible and effective MAT treatment models and integration approaches for primary care. ( $\mathrm{J}$ Am Board Fam Med 2020;33:512-520.)

Keywords: Buprenorphine, Combined Modality Therapy, Naltrexone, Narcotic Antagonists, Opiate Substitution Treatment, Opioid-Related Disorders, Primary Health Care, Psychotherapy, Qualitative Research, Virginia

\section{Introduction}

Drug overdose deaths involving opioids continue to rise in the United States, with 67,367 individuals fatally overdosing in 2018. ${ }^{1}$ Yet, most of these individuals are not receiving treatment in the substance use treatment system. ${ }^{2}$ Although integrating medicationassisted treatment (MAT) into primary care settings is proven to expand access to opioid use disorder (OUD) treatment, there has been limited diffusion and adoption of relatively new treatments (eg,

This article was externally peer reviewed.

Submitted 29 June 2019; revised 14 February 2020; accepted 21 February 2020.

From the Department of Family Medicine and Population Health, School of Medicine, Virginia Commonwealth University (EMB, ST).

Funding: The authors received support from the Virginia Department of Medical Assistance Services as part of Virginia Commonwealth University's independent evaluation of the ARTS benefit.

Conflict of interest: none.

Corresponding author: E. Marshall Brooks, PhD, 830 E. Main Street, Richmond, VA 23219 (E-mail: edward. brooks@vcuhealth.org). buprenorphine and injectable naltrexone) that could easily be adapted to community-based settings. ${ }^{3,4}$ The Drug Addiction Treatment Act, passed nearly 2 decades ago, allows office-based clinicians to prescribe buprenorphine; yet, there has been very little integration of OUD treatment in primary care practices. ${ }^{5}$

The atrophied state of addiction treatment-in addition to the fact that most family physicians receive no training in addiction ${ }^{6}$ - has led to fewer than half of the 2.2 million people who need treatment for opioid addiction actually receiving it. ${ }^{7}$ Also, despite that prescription opioids contribute to nearly $40 \%$ of overdose deaths, fewer than $4 \%$ of the 900,000 US physicians who can prescribe opioid painkillers have become licensed to prescribe buprenorphine to treat people with OUD. ${ }^{8}$

Despite a slow uptake, integrating MAT into primary care is seen as a paramount strategy for combating the opioid epidemic. Previous research shows that MAT in primary care can enhance access to treatment, reduce costs, improve patient experiences of care, and improve patient outcomes. ${ }^{9}$ 
Primary care-based MAT may particularly benefit low-resource patients who often struggle to access and maintain successful engagement with the substance use treatment system. By integrating addiction treatment services into primary care, practices can better identify and address patients' substance use issues, as well as co-occurring physical and mental health issues. ${ }^{10}$

Although numerous resources are available to aid primary care physicians in getting buprenorphine waivers, there is little research on the ways in which primary care practices are integrating and operationalizing MAT models. ${ }^{11-14}$ This article describes common goals related to integrating MAT at 26 outpatient clinics, strategies to operationalize those goals, and challenges related to integrating MAT for OUD into primary care.

\section{Methods}

Virginia implemented the Addiction and Recovery Treatment Services (ARTS) program in April 2017 to increase access to treatment for Medicaid members with opioid or other substance use disorders (SUDs). Seeking to expand access to OUD treatment in outpatient settings, the ARTS benefit allows providers to apply for "Preferred Office-Based Opioid Treatment" (P-OBOT) status. Attaining a P-OBOT status allows buprenorphine-waivered providers to prescribe buprenorphine products without submitting prior authorization requests and provides higher reimbursements for various services.

The Virginia Department of Medical Assistance Services, which runs the Medicaid program, contracted with Virginia Commonwealth University School of Medicine to conduct an independent evaluation of the ARTS program. The evaluation was conducted by faculty and staff from the Department of Health Behavior and Policy and the Department of Family Medicine and Population Health. Information was collected through semistructured interviews with 26 P-OBOT clinics in various regions and settings across Virginia. All clinics that had applied for POBOT status by April 2018 were invited to participate. Further details about the ARTS program and Virginia Commonwealth University's evaluation are available in earlier publications. ${ }^{15}$

\section{Interviews}

We conducted hour-long, group phone interviews with P-OBOT team members at all 26 sites. Group interviews minimally included a buprenorphinewaivered prescriber and a behavioral health $(\mathrm{BH})$ provider. Nurses, care managers, and peer counselors were also involved in the interviews, when available. Each clinician or staff member was asked about the aspects of the P-OBOT model or clinic operations with which they were most involved. Interviews were intended to elicit information concerning implementation experiences and clinic models, so practice or health system administration personnel not directly involved in clinic organization or patient care were not included in the sample. Interviews were semistructured and included questions about the overall clinic organization and structure, history of offering substance use treatment services, MAT implementation experiences, medical management practices, $\mathrm{BH}$ counseling, care coordination services, patient population characteristics, and ongoing needs and challenges. All interviews were audio recorded and transcribed. The project team also conducted 1-day site visits at 2 of the P-OBOT clinics, which included a tour of the clinic facilities, conversation with practice leaders and staff, and collection of practice tools and documents.

\section{Data analysis}

We used the qualitative analysis software Atlas.ti to organize and store qualitative data from the semistructured interviews and site visits. Data included interview transcripts and site visitor notes. We then used template-based and emergent coding techniques to thematically analyze our qualitative data, drawing on both a priori and emergent codes. ${ }^{16-19}$ An a priori codebook was created using pieces of the Consolidated Framework for Implementation Research to identify how characteristics of the OBOT model, the internal practice setting, the external setting, and individual clinicians appeared to help or hinder MAT integration. The a priori codebook also reflected prominent issues around MAT integration found in peer-reviewed and gray literature. During coding, emergent themes were identified and added to the codebook. We then followed a protocol-driven approach to analysis that included the following: (1) group reading of the data to refine a priori codes, identify emergent codes, and reach an agreement on code definitions; (2) independent test coding, during which a subset of documents, selected for variation, were coded to test the operational limits of the codebook and the 
ability of coders to apply codes reliably and consistently; and (3) independent coding combined with scheduled merges of coded data and weekly team coding huddles to allow for early detection of threats to intercoder reliability.

\section{Results}

The 26 P-OBOT clinics represent a range of organization types, sizes, and settings across Virginia (Table 1). Roughly $75 \%$ of the sites had been offering some form of SUD treatment services before the ARTS benefit went into effect. Pre-ARTS treatment options at these sites largely focused on nonpharmacological, $\mathrm{BH}$-focused counseling services. However several privately owned sites with buprenorphine-waivered physicians on staff also offered MAT or functioned as intensive outpatient programs. Previous efforts to expand SUD treatment were deemed logistically and/or fiscally unsustainable. With enhanced reimbursement rates, the ability to bill for care coordination services and peer support, and the elimination of prior authorization requirements, the ARTS benefit provided the necessary motivation to begin offering MAT. Findings and themes, along with example quotations, are summarized in Table 2.

Table 1. Clinic Characteristics

\begin{tabular}{lr}
\hline Characteristic & $\mathrm{N}(\%)$ \\
\hline $\begin{array}{l}\text { Location* } \\
\text { Rural }\end{array}$ & $6(23.1)$ \\
$\quad$ Urban & $20(76.9)$ \\
Number of medical providers & \\
1 & $9(34.6)$ \\
2 to 5 & $11(42.3)$ \\
$>5$ & $6(23.1)$ \\
Number of behavioral health clinicians & \\
1 & $2(7.7)$ \\
$2-5$ & $13(50.0)$ \\
$>5$ & $11(42.3)$ \\
Organization type & \\
Community service board & \\
Private practice & $13(50.0)$ \\
Health System-owned practice & $8(30.7)$ \\
\hline
\end{tabular}

*The Rural Urban Continuum Code (RUCC) was used to categorize each clinic as rural or urban. Urban was defined as having a RUCC of 1-3 and rural as having a RUCC of 4-9.

${ }^{\dagger}$ Community service boards are county- or city-run clinics mandated by Virginia code that provide behavioral health, developmental disability, and primary care services.

\section{Initial implementation of MAT}

Most sites reported relatively unchallenging implementation experiences. This was especially the case for federally qualified health centers, community service boards, and other privately owned SUD recovery clinics that already had robust $\mathrm{BH}$ infrastructures in place or were well integrated into larger health systems with established referral networks, which partially explains their implementation success. For such sites, the first several months of implementation consisted of fine-tuning clinic workflow and team member responsibilities, as well as adapting to the new fee structures and billing codes. Relatedly, these sites seemed to benefit from having clinical team members already on staff who had previous interest or experience in SUD treatment. For some sites, electronic health systems had to be reprogramed to include new P-OBOT-specific codes.

Identifying, hiring, or retaining team members was also a first priority. Rural clinics reported difficulty recruiting buprenorphine-waivered prescribers and $\mathrm{BH}$ clinicians. One site reported high turnover among $\mathrm{BH}$ clinicians, saying that once clinicians received their license, they left to pursue private practice where they could receive higher salaries. Long travel distances further hampered rural sites' ability to recruit MAT clinicians. To overcome this challenge, 2 sites created telehealth arrangements with prescribers to see patients virtually. One site recruited a physician board certified in addiction medicine to see patients 1 day a week via teleconference and drive into the clinic ( 3 hours round trip) for in-person visits once a month.

\section{Patient selection and exclusion criteria}

Most sites reported seeing a diverse mix of patients with a wide range of demographic characteristics, health care needs, and social challenges. In general, sites reported seeing slightly more men than women. Several sites reported being open to pregnant women, but 1 reported that they often end up having to refer these patients out for more specialized treatment. Two sites facilitated women-only programs, primarily for pregnant or postpartum women.

Patients with comorbid mental health diagnoses were extremely common, particularly those with attention deficit disorders, posttraumatic stress disorder, bipolar disorder, borderline personality disorder, and anxiety disorder. Clinics reported that 
Theme 1: Mixed implementation experiences

Findings:

Clinics that already had BH infrastructure, such as FQHCs, and

Example quotes:

CSBs had an easier time with implementation.

Clinicians credited the ARTS benefit for empowering them to address the opioid epidemic in their communities.

Practices struggled to recruit buprenorphine-waivered prescribers and $\mathrm{BH}$ clinicians willing and/or credentialed to work in a P-OBOT clinic, especially in rural areas.

"When I started learning about the ARTS initiative, I educated the administration on the changes that were coming down the pike. I knew I would want to leverage the ARTS initiative to expand services."

"Our doctor [...] actually lives in Maryland. She does it via telehealth."

Theme 2: Stigma from local communities and other health care providers

Findings:

Stigma towards MAT in local communities impacted sites' implementation experiences and clinical decision making, especially in rural areas.

Health care provider stigma, which was articulated about the addictive potential and relative harm of buprenorphine resulted in barriers to expanding MAT.Community engagement efforts helped alleviate community opposition.
Example quotes:

"We had to really try to define how we can provide the service without it becoming something the community says, 'we don't want this here anymore."”

"A lot of them will never understand. . why I'm not just trading one drug for another. Addiction is ending up in jail and not buying food for your kids, and injecting drugs into your penis and getting endocarditis in your heart valves. These people aren't doing that with Suboxone."

Theme 3: High prevalence of co-occurring medical, psychological, and social challenges.

\section{Findings:}

Comorbid mental health diagnoses and polysubstance use extremely common.

Housing and employment issues were common, especially in rural areas.Poor social stability resulted in challenges with outpatient OUD treatment.

Theme 4: Various approaches to induction and stabilization

Findings:

At-home inductions primarily were used to accommodate staff schedules rather than for medical needs.

Although most clinics responded to relapses and polysubstance use initially with increasing intensity of care, clinics have different thresholds for dismissal and/or referral to higher levels of care.

Theme 5: Behavioral health as "key to success"

Findings:

Group counseling the most widely used behavioral health intervention.Group therapy believed to provide necessary social support and reinforce change in socialization patterns. Many sites offered multiple session times and treatment modalities to promote patient engagement in group therapy.

Example quotes:

"One of our patients was essentially homeless when she started with us, but wasn't really considered homeless by our local DHS standards because she was sleeping on her friend's couches, but she might sleep on three different couches a week."

\section{Example quotes:}

"Our doctor works for us a day a week, so we have to do inductions offsite. I don't have any options for doing them onsite."

"If we have a patient relapse, our model of care is, you need to have additional services."

Example quotes:

"Medication assists the therapy rather than the other way around."

"You watch this dynamic where they develop true genuine feelings for each other, like real concern. They'll talk about, 'you know what keeps me sober, is I don't want to let this group down."”

"[Group therapy] is where the healing happens."

Theme 6: Large variation in care coordination structure and intensity

Findings:

Large variation in the goals and intensity of care coordination efforts, as well as primary means of identifying patients' cooccurring needs.

The majority of clinics used a team approach to care coordination rather than having dedicated care coordinators.

Enhanced care coordination was facilitated by weekly or biweekly meetings to facilitate communication, information sharing and strategic planning.

Theme 7: Peer support services highly viewed but inconsistently implemented

\section{Findings:}

Peers described as better able to provide personal support to patients, help patients manage cravings or stress, and connect patients to social support networks in the community.

Only one-third of the sites used peer support services.
Example quotes:

"When you're drowning in a sea of addiction, we're going to be the life boat you want to hold onto." We always make Monday lunchtime meetings, all of us, the whole treatment team, and we review every patient that's in need of review."
Example quotes:

"We, unfortunately, are bumping up against [barriers] trying to add peer recovery coaches. You have to present your business plan and how you're going to cover expenses."

"We've found with the peer recovery coach that patients feel a connection because this person has kind of been there, done that." 
Theme 8: Treatment success ambiguous to define and difficult to measure

Findings:

Successful/stable patient largely defined in functional terms, as a change in physical appearance, social relationships, and ability to hold a job.

Sites expressed desire for measures through which to assess and compare themselves to other sites.

Tapering off buprenorphine not seen as necessary for "successful treatment."

Attrition at some sites may be due to treatment models requiring 3 or more weekly appointments, especially for sites operating in rural, outlying areas.

Theme 9: Complexity best met with collaboration, compassion, and consistency

Findings:

Strong collaborative relationships between medical, $\mathrm{BH}$, and care coordination team members emphasized as the most important factor in effectively addressing patients' complexly interconnected medical and social needs.

Maintaining compassionate, consistent and adaptive approach seen as the key to creating therapeutic relationships with patients.
Example quotes:

"Success is always functional. . somebody whose disease

(including psychiatric comorbidities) is managed."

"You can see it in their eyes, in their face, in their demeanor."

"... not necessarily coming off of [buprenorphine] because I

know very few people who have. Some of them try to come off and it doesn't work. They find themselves right back where they started. So, that's what we want to avoid."

$\mathrm{BH}$, behavioral health; FQHC, federally qualified health center; CSB, community service board; MAT, medication-assisted treatment; P-OBOT, Preferred Office-Based Opioid Treatment; ARTS, Addiction and Recovery Treatment Services; DHS, Department of Homeland Security; OUD, Opioid Use Disorder.

patients with borderline personality disorder or attention deficit hyperactivity disorder were the most challenging and often were the least successful in treatment due to impulse control and emotional volatility issues. As a result, some clinics specifically excluded these patients or patients with comorbid serious mental illness and instead referred them to more intensive outpatient programs. Nonopioid SUDs and polysubstance use were also extremely common. Methamphetamine use was particularly prevalent in the southwest region of Virginia where its wide availability and ability to mitigate the lethargy and anhedonia often experienced during recovery made it attractive to patients.

When asked about exclusion criteria, 15 (50\%) clinics made no mention of excluding patients who use benzodiazepines, despite regulations specifying their ineligibility for treatment. Only 5 clinics stated that patients using benzodiazepines would be ineligible or required to go through detoxification before beginning treatment. Several sites reported high rates of patients entering treatment while already on illicit buprenorphine. Hepatitis $\mathrm{C}$ was the most frequently reported comorbid medical condition.

Sites reported widespread social challenges. Housing and employment issues were most common but often the most intractable. A lack of housing support for women was especially challenging, as this population often entered treatment while
Example quotes:

"It's kind of like it's all hands on deck kind of thing. Where we all kind of take it, but one of the benefits has been that we do have a lot of dual diagnosed clients who are seeing another therapist and who are seeing a support coordinator or seeing a psychiatrist so a lot of their issues are being taken care of in house, which provides the stability, but we collaborate." living in domestic abuse situations. Inadequate or unpredictable access to transportation services similarly hampered patients' ability to maintain treatment, especially in rural areas. Several sites preferred patients with stable housing and transportation and a working cell phone, as such patients were considered better suited for outpatient treatment.

\section{Engaging communities and combating stigma}

Roughly half of the P-OBOTs encountered hostility toward MAT in their local communities. One site told us, " 1 of the concerns with Suboxone is if you bring it into the community, you are also bringing in drug addicts, or people are going to come running for it and then become drug addicts when the reality is they are already in the community." Such stigma toward MAT is not benign. It directly and indirectly affects implementation experiences and clinical decision making. For example, community pushback from local residents and city officials compelled 1 site to shift from a harm reduction model, in which small amounts of cannabis or prescribed stimulants were tolerated in the program, to a policy of zero tolerance. In such small rural communities, negative public perceptions threaten to discourage patients with OUD from using MAT services and/or persuade regular primary care patients to seek care elsewhere. "We had 
to really try to define how we can provide the service without it becoming something the community says, 'we do not want this here anymore." At this site, such infractions are now grounds for dismissal from the program and automatic referral to a higher level of care. Many patients at this site reportedly could not afford the indirect costs of engaging in higher levels of treatment to which they were referred or were delayed access for weeks or months due to chronically limited service capacities. Also, since becoming a P-OBOT clinic in late 2017, this site has been forced to "close out" a large portion of their patient population due to this policy of zero tolerance.

Although stigma toward MAT was pervasive at the community level, sites spoke at greater length about stigma from fellow clinicians and staff members. For example, we were frequently told about colleagues who described MAT as "just another addiction." At several sites, this view of MAT contributed to some clinicians being hesitant about or outright refusing to participate in MAT, largely on ideological grounds. For other colleagues, refusing to offer MAT was instead fueled by an intolerance toward "those patients" being introduced into "my clinic," as we often heard it described. For example, 1 site wanted to create their own version of the "Vermont hub and spoke model," in which patients who had been induced on buprenorphine at 1 site and were now stable would transfer to another primary care setting for medication maintenance. However, senior administrators reportedly refused to participate in the initiative, saying they did not want to be perceived as "the kind of office" that takes "those kinds of patients." Such misunderstanding about MAT and the populations they serve reflect both a lack of knowledge about the evidence for and benefits of pharmacological interventions, as well as a more profound ignorance regarding the nature of addiction.

In an attempt to mitigate stigma against MAT, several sites engaged in various forms of community engagement. This included making educational materials on MAT publicly available and actively distributing them to community leaders; speaking about and offering workshops on MAT in schools, churches, and other community centers; serving on community coalitions and leadership committees; personally meeting with public officials, including local elected figures, judges, and law enforcement; and talking with Narcotics Anonymous groups (who are still largely opposed to MAT). Combatting internal forms of stigma from fellow clinicians and staff largely took the form of providing continuing education on the strategy and benefits of MAT during clinic meetings and indirectly addressing latent biases by internally celebrating treatment successes. Sites that reported participating in community outreach also reported being less impacted by community stigma.

\section{Patient intake and medication management}

Sites uniformly reported conducting thorough intake procedures, including screening for OUD and other polysubstance use, taking detailed medication histories, diagnosing any co-occurring medical or mental conditions, and documenting social concerns that may impact patients' ability to participate in MAT.

Sites varied on their approach to induction-the medically monitored startup of buprenorphine treatment-with roughly twice as many sites conducting in-clinic inductions as opposed to at-home inductions. Those that preferred at-home inductions, in which patients self-administer initial doses of buprenorphine until cravings are adequately controlled, seemed to do so primarily to accommodate prescriber schedules. After intake, medical visits were primarily used to discuss the effectiveness of medication at controlling cravings; any necessary changes in dosage or side effects; and other cooccurring medical, social, or mental health concerns.

Medication visits routinely occurred the same day as BH counseling. Most sites held 1-on-1 medication visits, with the exclusion of 1 site, whose nurse practitioner consulted with patients during group visits. During medication visits, patients were also subjected to urine drug screens (UDSs) and/or random pill/wrapper counts. However, there was some variability in how often a UDS was collected, the supervision of UDSs, and whether sites routinely sent samples out for testing once collected. Positive UDSs were reportedly grounds for dismissal at 3 sites. Most sites instead responded to relapses or other polysubstance use with increases in the number of required group counseling sessions, the addition of individual therapy (if not already required), and decreases in prescription times. Interestingly, 1 site reported dealing with chronically recalcitrant patients by inviting family members to attend counseling sessions, and another 
decreased buprenorphine doses until patients adequately participated in counseling. Those patients requiring elevated levels of treatment were referred to specialty MAT services, such as methadone treatment providers or residential treatment facilities, where and when available. However, in rural areas of Virginia, such services are frequently inaccessible due to long travel distances for patients or extended wait times.

\section{BH counseling}

$\mathrm{BH}$ counseling was primarily offered in groups, where social support was believed to lead to a change in "socialization patterns." For example, 1 $\mathrm{BH}$ clinician described regularly witnessing collective transformations in peoples' interactions, attitudes, and motivations after a few weeks of group visits. "You watch this dynamic where they develop true genuine feelings for each other, like real concern. They will talk about, "you know what keeps me sober, is I do not want to let this group down."” Similarly, 1 physician stated that "Medication assists the therapy rather than the other way around."

To facilitate participation in group therapy, sites offered multiple sessions throughout the week that patients could choose from. One site reportedly held 13 sessions a week. The format of group sessions similarly varied considerably from site to site. Some sites used formal curricula, such as the "Living and Balance" curriculum, or used group sessions primarily for patient education on topics such as craving management strategies, sleep hygiene, or nutrition. Other sites attempted to offer patients a variety of therapy strategies and approaches, including Narcotics Anonymous-style sessions, groups featuring guest speakers, dialectical behavioral therapy groups, groups focused on specific mental health issues, and gender-specific groups-particularly for women-focused on challenges related to sexual trauma, domestic abuse, or parenting. Four sites reported more free-form approaches to group sessions in which self-reflection and interpersonal support took precedence over "educational" didactics or other treatment modalities.

Most sites also offered individual counseling. Although individual counseling was required for at least some portion of clinics' treatment models, individual counseling was primarily offered to patients with anxiety or trauma-related issues, where group settings were either intimidating or undermined by more deep-seated mental health issues. Several sites stated plans to offer long-term individual counseling to patients for whom recovery from an opioid addiction had uncovered emotional traumas needing more intensive mental health treatment.

\section{Care coordination}

Care coordination aimed to address patients' often numerous co-occurring medical, mental health, and social challenges by facilitating connections to additional health care or community-based services. This included primary care (if the patient was not receiving primary care services at the P-OBOT site), specialized treatment for serious mental illnesses, housing support, employment services, and transportation assistance.

The goals and intensity of care coordination efforts varied considerably. Most sites used intake assessments to identify co-occurring needs at the outset of treatment and then passively identified emergent issues during medication visits, group therapy, and individual counseling sessions. A select few required patients to regularly meet with care coordinators to actively screen for emergent needs and track progress. One site delegated all care coordination responsibilities to peer recovery specialists, referring to them as the patients' primary "coach" or "office sponsor." Two sites had extraordinarily dedicated care coordinators who stayed in daily contact with patients, largely via text messaging, to monitor progress and intervene on emerging issues.

Conversely, roughly two-thirds of the sites approached care coordination as a "team effort." Team communication was facilitated by Electronic Health Record messaging and weekly or biweekly meetings to ensure timely information sharing and to coordinate care plans. Regular meetings also allowed teams to preemptively intervene on emerging issues, connect patients to community resources, and maintain a "united front" when patients were having challenges.

\section{Peer support services}

Roughly one-third of the sites we spoke to reported offering peer support services. Peer counselors participated in group sessions, offered individual assistance, or assisted with care coordination. One site even delegated all care coordination responsibilities to peer recovery specialists, referring to them as the 
patients' primary "coach" or "office sponsor." This site credited the peer support coaching program for improving retention rates. Peers were often credited with offering personal support to patients, and were found to be particularly effective at "building connections" with patients; "talking patients down" when cravings, anxiety, or stress became too high; and connecting patients to trusted community support networks.

\section{Assessing treatment success}

We routinely asked sites about how they measured treatment success. Although some sites described monitoring the number of group visits and individual counseling sessions a patient attended or number of days since a positive UDS, few sites used discrete metrics for measuring success or counted the number of "successful" patients. But, rather than indicating ineffective treatment or inaccurate record keeping, this likely reflects the complexity of concepts like "success" or "recovery" in these treatment settings. Most sites, therefore, instead relied on indications of improved functional status. One site described it this way: "Success is always functional... somebody whose disease (including psychiatric comorbidities) is managed." Another suggested that "stability" is evidenced in a patient's physical appearance, social relationships, and ability to hold a job, saying that "You can see it in their eyes, in their face, in their demeanor."

Only 2 sites required patients to taper: one because the prescribing physician believed it perpetuated patients' addictions and the other because $\mathrm{BH}$ clinicians believed addiction could be overcome through personal discipline. However, most sites saw indefinite maintenance as an acceptable, and often necessary, part of recovery. For example, 1 site explained that "Success is maintaining a healthy, happy, productive life on the lowest amount of Suboxone possible. But not necessarily coming off of it because I know very few people who have. Some of them try to come off and it does not work. They find themselves right back where they started. So, that is what we want to avoid."

Sites reported several keys to success. Of particular importance are strong collaborative relationships between medical, $\mathrm{BH}$, and care coordination team members. Although all P-OBOT sites are required to use interprofessional teams, simply colocating or coordinating services is often insufficient. Instead, a heightened sensitivity to each patient's unique needs and challenges and more intentionally incorporating multimodal treatment strategies is the "key to success." Sites also emphasized the importance of maintaining compassionate, consistent, and adaptive relationships with patients receiving MAT: that is, a humanistic sensitivity to patients, a commitment to monitor and enforce adherence to program requirements, and a willingness to accommodate and adapt to patients' unique needs.

\section{Discussion}

Our study identified various ways in which MAT can be implemented in primary care clinics and other ambulatory settings. Although state regulations and treatment guidelines suggest colocating $\mathrm{BH}$ counseling and medication management, providing care coordination, and conducting regular UDSs, we found a wide spectrum of ways in which practices can adapt and innovate treatment models to fit local needs.

Although initial results are promising, the longterm viability of the $\mathrm{P}-\mathrm{OBOT}$ model in primary care will require identifying sustainable staffing solutions and addressing deep-seated community distrust of MAT and people with OUD. Although we found that an inadequate supply of qualified clinicians was a challenge to many practices in Virginia aiming to maintain or expand services, as viable treatment models are developed and disseminated, the number and availability of clinicians may increase as well. In the meantime, practices are taking advantage of adjunctive staffing solutions, including the use of telehealth and team-based treatment models.

Stigma toward MAT and people with SUDs remains a challenge, particularly for rural primary care clinics. Regions most deeply scarred by the opioid epidemic and most lacking in health care services also seem the wariest of welcoming MAT programs into their communities. Yet, practices across the country have begun countering these community concerns by actively engaging in education strategies and positive messaging about SUDs and treatment options.

Our study has several limitations. Our findings reflect themes we observed in the $26 \mathrm{P}-\mathrm{OBOT}$ practices, which were the first to adopt the P-OBOT model in Virginia and may, therefore, reflect more progressive practices. In addition, we did not obtain data on treatment outcomes, so we are unable to assess the effectiveness of the P-OBOT practices' 
MAT integration models. In addition, we do not have a comparison group, so we are not able to assess the extent to which MAT integration activities at our sites differed from other primary care practices working to integrate MAT services.

The authors thank the Department of Medical Assistance Services for providing their technical expertise on the Medicaid claims data and the ARTS program. The conclusions in this article are those of the authors, and no official endorsement by the Virginia Commonwealth University School of Medicine or Virginia Department of Medical Assistance Services is intended or should be inferred.

To see this article online, please go to: http://jabfm.org/content/ 33/4/512.full.

\section{References}

1. Hedegaard H, Miniño AM, Warner M. Drug overdose deaths in the United States, 1999-2018. NCHS Data Brief, no 356. Hyattsville, MD: National Center for Health Statistics; 2020.

2. Substance Abuse and Mental Health Services Administration. Key substance use and mental health indicators in the United States: Results from the 2018 National Survey on Drug Use and Health (HHS Publication No. PEP19-5068, NSDUH Series H-54). Rockville, MD: Center for Behavioral Health Statistics and Quality, Substance Abuse and Mental Health Services Administration; 2019.

3. Roman P, Abraham A, Knudsen H. Using medication-assisted treatment for substance use disorders: Evidence of barriers and facilitators of implementation. Addictive Behav 2011;36:584-9.

4. Cicero TJ, Ellis MS, Surratt HL, Kurtz SP. The changing face of heroin use in the United States: a retrospective analysis of the past 50 years. JAMA Psychiatry 2014;71:821-6.

5. Vestal C. In fighting an opioid epidemic, medication-assisted treatment is effective but underused. Health Aff 2016;35:1052-7.

6. Tong S, Sabo R, Aycock R, et al. Assessment of addiction medicine training in family medicine residency programs: a CERA study. Fam Med 2017;49:537-43.

7. Sullivan $\mathrm{P}$. White House to request $\$ 1$ billion to counter drug abuse epidemic. The Hill [serial on the internet]. Available from: https://thehill.com/ policy/healthcare/267895-white-house-proposes-1billion-to-fight-opioid-epidemic. Published 2016.
8. National Center for Health Statistics Division of Vital Statistics. Mortality data. Available from: http:// www.cdc.gov/nchs/deaths.htm. Published 2018. Accessed 27 July, 2018.

9. Jones C, Campopiano M, Baldwin G, McCanceKatz E. National and state treatment need and capacity for opioid agonist medication-assisted treatment. Am J Public Health 2015;105:e55-63.

10. LaBelle CT, Han SC, Bergeron A, Samet JH. Officebased opioid treatment with buprenorphine(OBOTB): statewide implementation of the Massachusetts Collaborative Care Model in community health centers. J Subst Abuse Treat 2016;60:6-13.

11. Dowell D, Haegerich TM, Chou R. CDC guideline for prescribing opioids for chronic pain-United States, 2016. JAMA 2016;315:1624-45.

12. Lembke A, Humphreys K, Newmark J. Weighing the risks and benefits of chronic opioid therapy. Am Fam Physician 2016;93:982-90.

13. American Academy of Family Physicians. AAFP chronic pain management toolkit. Available from: https://www.aafp.org/patient-care/public-health/ pain-opioids/cpm-toolkit.html. Published 2018. Accessed 27 July, 2018.

14. National Institute on Drug Abuse. Screening and Assessment Tools Chart. Available from: https://www. drugabuse.gov/nidamed-medical-health-professionals/ tool-resources-your-practice/screening-assessmentdrug-testing-resources/chart-evidence-based-screeningtools. Published 2018. Accessed 27 July, 2018.

15. Cunningham P, Barnes A, Sheng Y, et al. Addiction and recovery treatment services. Access and utilization during the first year (April 2017-March 2018). An evaluation report prepared for the Virginia Department of Medical Assistance Services. Available from: http://www.dmas.virginia.gov/files/links/1625/ ARTS\%20one-year\%20report\%20(08.09.2018).pdf. Published 2018.

16. Gibbs G. Analyzing qualitative data. London, England: SAGE Publications, Ltd; 2007.

17. King N. Qualitative organizational research: coremethods and current challenges. London, England: Sage Publications, Inc.; 2012.

18. Braun V, Clarke V. Using thematic analysis in psychology. Qual Res Psychol 2006;3:77-101.

19. Crabtree BF, Miller WL. Using codes and codemanuals: a template organizing style of interpretation. In: Miller BF, Crabtree WL, editors. Doing qualitative research, 2nd ed. Thousand Oaks, CA: Sage Publications; 1999. P. 163-77. 